\title{
Variability in billing practices for whole-body magnetic resonance imaging
}

\author{
Andrew J. Degnan ${ }^{1,2}$ (D) Mark D. Alson ${ }^{3} \cdot$ Richard Duszak Jr. $^{4}$ \\ Received: 31 July 2018 / Accepted: 30 August 2018 / Published online: 7 September 2018 \\ (C) Springer-Verlag GmbH Germany, part of Springer Nature 2018
}

Dear Editors,

The recent article by Schooler and colleagues [1] offers new and useful insight into the utilization and reporting of pediatric whole-body MRI across many institutions. The authors highlighted several areas for opportunity to advance the practice of whole-body MRI, which encompasses many valuable clinical indications. It is important to note, as the authors did, that substantial variability exists in the technical parameters, reporting and billing of whole-body MRI.

We have been actively engaged in Current Procedural Terminology (CPT) code development in radiology and read with interest their findings about how radiologists bill for these whole-body MRI services. In particular, the inhomogeneous reporting of pediatric whole-body MRI with either (1) separate chest, abdomen and pelvis codes or (2) an unlisted code leads to further confusion and could limit future studies examining utilization (e.g., how would such studies be unambiguously identified using claims data?). As the authors pointed out, no specific CPT exists to describe whole-body MRI. Depending on the study ordered, clinical indications, and technical parameters used, some whole-body MRI examinations might in fact include targeted evaluation, meriting reporting with dedicated chest, abdomen or pelvis

Andrew J. Degnan

degnana@email.chop.edu

1 Department of Radiology,

Children's Hospital of Philadelphia,

3401 Civic Center Blvd.,

Philadelphia, PA 19104, USA

2 Department of Radiology,

Perelman School of Medicine at the University of Pennsylvania,

Philadelphia, PA, USA

3 Sierra Imaging Associates, Clovis, CA, USA

4 Department of Radiology and Imaging Sciences, Emory University School of Medicine,

Atlanta, GA, USA examinations. On the other hand, we believe screening whole-body MRI might be more appropriately reported using an unlisted MRI CPT code (76498, unlisted magnetic resonance procedure [e.g., diagnostic, interventional]) [2]. Use of an unlisted code, however, could also necessitate prior authorization, depending on the payer, which should be considered in performing these studies and could prove an impediment to prompt patient access.

Extending the recommendations of Schooler et al. [1], we encourage pediatric radiologists to work with their coding and billing staff to ensure appropriate and accurate reporting. Radiologists and coders should work together to develop procedure codes that specifically track performance of whole-body MRI examinations within their institutions. Accurate tracking of these examinations has substantial importance for future assessment of clinical indications and utilization - and will be vital to capture necessary data should the CPT Editorial Panel consider dedicated codes for these services in the future. Wholebody MRI holds much promise for pediatric radiology and this recent article highlighted several key areas for future development to increase its applicability and standardize its performance and billing.

\section{Compliance with ethical standards}

Conflicts of interest None

\section{References}

1. Schooler GR, Davis JT, Daldrup-Link HE, Frush DP (2018) Current utilization and procedural practices in pediatric whole-body MRI. Pediatr Radiol 48:1101-1107

2. Chung CY, Alson MD, Duszak R Jr et al (2018) From imaging to reimbursement: what the pediatric radiologist needs to know about health care payers, documentation, coding and billing. Pediatr Radiol 48:904-914 